\title{
HLA-DR typing for kidney transplants: Advantage of polymerase chain reaction with sequence specific primers in a routine hospital laboratory
}

\author{
C Lynas, N J Hurlock, J A Copplestone, A G Prentice, R J S McGonigle
}

\begin{abstract}
Aims-To determine whether the polymerase chain reaction with sequence specific primers (PCR-SSP) can assign HLA-DR type more accurately than serology in a routine hospital laboratory. Methods-The 93 patients currently awaiting kidney transplants have been DR typed by serology over the past 14 years, $82 \%$ within the past five years. They have now been retyped using the PCR-SSP method described by Bein et al. Where the two results differed, PCRSSP was repeated, once by the same method and once using the primer set of Olerup and Zetterquist.

Results-There were 13 (14\%) discrepancies between the results. Of these, two were PCR-SSP failures, later overcome: three were failure to detect DRB1*0103 by serology; five assignment of other alleles by PCR-SSP to serological "blanks"; and three alleles were differently assigned by serology and PCR. The serological typing of the final patient when repeated for this study was at variance with the original findings (14 years ago), but in agreement with PCR. In the remaining patients, serology had not determined the split of $36 \mathrm{DR3}$ alleles (all DR17 by PCR-SSP) or 13 DR6 alleles (12 DR13 and one DR14 by PCR-SSP). One patient in each case had their antigen splits of DR2 and DR5 assigned by PCRSSP (DR15 and DR11, respectively) but not by serology.

Conclusions-PCR-SSP provides more reliable and detailed information on HLA-DR polymorphism than serology, and does so within a routine tissue typing laboratory.
\end{abstract}

(F Clin Pathol 1994;47:609-612)

Department of Haematology, Derriford Hospital, Plymouth PL6 8DH C Lynas

N J Hurlock

J A Copplestone

A G Prentice

Department of Renal Medicine

R J S McGonigle

Correspondence to:

Dr C Lynas

Accepted for publication

12 January 1994
Of the more than 130 HLA class II antigens currently recognised, ${ }^{1}$ those encoded by alleles at the DRB1 locus seem to be of particular importance with regard to graft rejection. ${ }^{2-4}$ Identification of these alleles has traditionally been by serology in a complement mediated microcytotoxicity assay. With the elucidation of the DNA sequences for the HLA genes, methods have been devised for genotyping patients. First among these defined the HLADRB1 polymorphisms by restriction fragment length polymorphism (RFLP), ${ }^{5}$ although not all genotypes can be resolved. ${ }^{6}$ Hybridisation of sequence specific oligonucleotide probes (SSOs) to genomic DNA can identify most known alleles, and in combination with the PCR (PCR-SSO), has become a sensitive and specific technique for genotypic analysis. ${ }^{7}$ Developments such as the reverse dot-blot ${ }^{8}$ and amplified fragment length polymorphism (AFLP) ${ }^{9}$ continue to be made.

All of these genotyping methods improve the confidence of allele assignment compared with phenotypic serology and permit more detailed identification of over 60 DRB 1 alleles now recognised. ${ }^{1}$ They do not depend on the purification of viable $B$ cells expressing the surface antigens and use easily obtainable reagents. However, they are time consuming to perform and some require sophisticated skills not yet widespread in routine tissue typing laboratories. PCR-SSP1011 is easier to interpret, is more specific, demands only simple and rapid post-amplification analysis, and for many heterozygotes it is more informative than other genotyping methods. Above all, the time it takes is comparable with that of serology. Thus it is likely to be most suitable for the routine laboratory. The procedure described by Bein $e t a l^{10}$ uses a nested PCR first to amplify exon 2 of the DRB genes and a DRw52 specific part of the DRB1 exon 2 . Amplification from these templates with a set of 18 nested primer pairs produces DRB1 allele specific products but no cross-reaction with the very similar sequences of other DRB genes. Olerup and Zetterquist ${ }^{11}$ use primers and a PCR cycling profile adjusted to obtain allele specific products in a single round of amplification.

There have been several reports ${ }^{12-15}$ which have compared the results of DR typing by serology and by restriction fragment length polymorphism (RFLP) or SSO. Some comparisons probably overestimate the true discrepancy rate because of the nature of the patient sample used. ${ }^{14}$ Others ${ }^{216}$ suggest $25 \%$ or more ${ }^{15}$ of alleles may be incorrectly assigned by serology. In some studies ${ }^{15}$ the serology was undertaken in routine laboratories, but the genotyping was only performed by highly trained experienced laboratories.

If PCR-SSP is to be the genotyping method of choice for the routine hospital laboratory, it must be shown to improve the accuracy of HLA typing compared with serology when used in such a laboratory. To this end we retyped by PCR-SSP all 93 patients awaiting kidney transplant in this non-teaching, subregional hospital. Although slower, we chose the method of Bein $e t a l^{10}$ as it had been optimised 
for a first generation thermocycler such as we had available. The success of the Olerup and Zetterquist ${ }^{11}$ PCR-SSP was said to depend on using an optimal amount of DNA (Savage D, et al. Abstract presented to 4th BSHI Annual Conference 1993). For staff inexperienced in DNA manipulation the system of Bein et al might thus prove more robust.

\section{Methods}

HLA-DR serology was undertaken by a standard two-stage microlymphocytotoxicity assay with the following modifications. Peripheral blood lymphocytes were enriched for B lymphocytes by passage through nylon wool columns (before September 1991) or with Dynabeads (Dynal Corporation). All patients' samples were tested using the UK Transplant Support Service Authority National HLA-DR typing set current at the time of presentation. All plates were independently read by at least two qualified MLSO staff and the type assigned by consensus. The patients were HLA-DR typed by serology at presentation but, with the exception of the longest standing patient initially typed in 1979 , have not been checked since. Most ( $82 \%$ ) of the patients were first seen within the past five years.

The PCR-SSP was conducted using the primer sets and nested amplification described by Bein et $a l^{10}$ in a Perkin Elmer 480 thermocycler. DNA was extracted from 0.5 $\mathrm{ml}$ of EDTA or citrated blood by a rapid mini-prep adaptation of the salting-out procedure described by Olerup and Zetterquist, ${ }^{11}$ and redissolved by eye to a concentration of $50-100 \mathrm{ng} / \mu \mathrm{l}$. The first round amplification (20 cycles) used $3 \mu \mathrm{l}$ of DNA per reaction. The products of these reactions were diluted 1 in 20 and $3 \mu$ l used as template in each second round amplification ( 30 cycles). The reaction mixes (total volume $26 \mu \mathrm{l}$ ) contained $0.25 \mu \mathrm{M}$ each of human growth hormone (HGH) primer, $0.5 \mu \mathrm{M}$ each of two allele specific primers, and $0.2 \mathrm{mM}$ each dNTP in $1 \times$ Taq reaction buffer (HT Biotechnology). After addition of the template and $30 \mu \mathrm{l}$ oil (melting point bath oil, Sigma), the tubes (thin walled PCR tubes, Anachem) were heated to $94^{\circ} \mathrm{C}$ for eight minutes before commencing cycling through $94^{\circ} \mathrm{C}$ for one minute, $65^{\circ} \mathrm{C}$ for 30 seconds, and $72^{\circ} \mathrm{C}$ for 30 seconds. Towards the end of the pre-heating period, $1 \mu \mathrm{l} \mathrm{Taq}$ polymerase (SuperTaq, HT Biotechnology), diluted 1 in 10 in $1 \times$ reaction buffer from a $-20^{\circ} \mathrm{C}$ stock already diluted 1 in 10 in $10 \times$ reaction buffer, was added. For analysis of the results, the aqueous phase was brought to the surface by centrifugation for one minute and $10 \mu \mathrm{l}$ electrophoresed on a $1 \%$ agarose (ICN)/TBE gel at $50 \mathrm{v} / \mathrm{cm}$ for about 20 minutes. Pre- and post-amplification samples were handled using separate, dedicated sets of pipettes to avoid risks of crosscontamination.

For all patients where results by the two methods were not in agreement, PCR-SSP was repeated, once by the same method and once using the Olerup primers. The latter method was performed using a primer kit supplied through The British Society for Histocompatibility and Immunogenetics (BSHI) exactly according to their protocol for $13 \mu \mathrm{l}$ reactions, except that cycling times (35 seconds at $94^{\circ} \mathrm{C}, 70$ seconds at $63^{\circ} \mathrm{C}$ ) were adapted for our first generation PCR machine.

\section{Results}

Comparison of results obtained by serology and PCR-SSP for the 93 patients revealed 13 (14\%) discrepancies (table 1).

Two of these were initial mistyping by PCR, but the problems causing these have subsequently been resolved. In case 1 PCRSSP suggested that the patient was homozygous DR4, while serology defined the additional presence of a DR6 allele. The apparent discrepancy between the results encouraged us to develop further the Bein PCR primer sets, ${ }^{17}$ so that we could detect the normally "undetectable" alleles DRB 1 ^1305, $\star 1404$, and $\star 1410$. The patient was found to carry the DRB1*1404 allele. The second mistyping by PCR (case 2) would have replaced a serological "blank" with a DR8 allele. We have subsequently noted that DRw17 patients frequently cause some degree of positive reaction with the DR8 primer set. PCR-SSP with the Olerup primers also suggested that this was a false positive.

Three patients (cases 3-5) were shown to carry the DRB $1^{\star} 0103$ allele by PCR but not by serology. These patients were all typed before the provision of $\mathrm{DRB} 1^{\star} 0103$ antiserum on the typing plates used.

Five patients (cases 6-10) were recorded as homozygous by serology but were shown to be heterozygous by PCR-SSP. The discrepancies can be explained as follows. Because it was impossible to define the presence of DR12 in the presence of DR11 by serology, case 6 was recorded as homozygous. Cases 7 and 9 were first typed in 1984 and 1990, respectively, before assignment of DR9 was reliable using serology. The DR6 allele was missed by serology in case 8 because there

Table 1 Discrepancies in DRB1 type between serology and PCR-SSP

\begin{tabular}{|c|c|c|}
\hline Case No & Serology & $P C R-S S P$ \\
\hline \multicolumn{3}{|c|}{ Initial $P C R$ failure } \\
\hline $\begin{array}{l}1 \\
2\end{array}$ & $\begin{array}{l}\text { DR4, DR6 } \\
\text { DR3, DRw52 }\end{array}$ & $\begin{array}{l}\text { DR4, -^ } \\
\text { DRw17, DR8 }\end{array}$ \\
\hline \multicolumn{3}{|c|}{ Allele missed by serology } \\
\hline 3 & DR1, - & $\mathrm{DR} 1, \mathrm{DRB} 1{ }^{\star} 0103$ \\
\hline 4 & DR7, - & $\mathrm{DR} 7, \mathrm{DRB} 1{ }^{*} 0103$ \\
\hline 5 & DR3, - & DRw17, DRB 1*0103 \\
\hline 6 & DRw11, - & DRw11, DRw12 \\
\hline 7 & DR4, - & DR4, DR9 \\
\hline 8 & DR4, - & DR4, DRw13 \\
\hline 9 & DR1, - & DR1, DR9 \\
\hline 10 & DR4, - & DRw15, DR4 \\
\hline \multicolumn{3}{|c|}{ Allele wrongly assigned by serology } \\
\hline 11 & DR4, DR6 & DR4, - \\
\hline 12 & $\mathrm{DR} 1, \mathrm{DR} 9$ & DR1, DR4 \\
\hline 13 & DR3, DR6 & DRw16, DRw17 \\
\hline
\end{tabular}

* Devising new primer combinations to detect otherwise undetectable alleles revealed this blank was in fact DRB1 1404 . + Patient was PCR negative with both the normal Bein prime 
Table 2 Allele splits not assigned by serology

\begin{tabular}{cll}
\hline & Serology & PCR-SSP \\
\hline 1 patient & DR2, x & DRw15, x \\
1 patient & DR5, DR6 & DRw11, DRw13 \\
30 patients & DR3, x & DR17, x \\
7 patients & DR6, x & DR13, x \\
1 patient & DR6, x & DR14, x \\
4 patients & DR3, DR6 & DRw17, DRw13 \\
1 patient & DR3, ?DR6 & DRw17, DRw13 \\
2 patients & DR3, y & DRw17, z \\
\hline
\end{tabular}

Where $\mathbf{x}$ is second allele on which the two methods agreed. Where $y$ and $z$ were second alleles on which the two methods did not agree.

was only a weak reaction to the antisera, possibly because of poor B cell purity (typing was done before the use of Dynabeads). The failure to detect DR2 in case 10 is attributed to the date-1983 - of the original serological typing.

The final three discrepancies (cases 11-13) were due to the wrong assignment of alleles by serology. Of these, case 11 had been originally typed 14 years ago and never rechecked. On repeating the serology this year, in response to PCR findings, results were at variance with the patient's reported DR type but in concordance with PCR findings. Case 12 gave only weak reactions with the DR4 antisera, most of which contain weak DR9 reactivity. He was therefore wrongly defined as DR9. Serological DQw 1 reaction suggested that case 13 was not homozygous DR3. On this basis DR6 rather than DR2 was assigned as most DR2 antisera are DRw15 with only weak DRw16 activity.

Positive results for the alleles assigned by PCR were also obtained using the Olerup and Zetterquist primers for patients where blanks or different alleles had been assigned by serology.

PCR-SSP frequently provided more detail than serology, where broad antigen splits are often all that can be defined. With two exceptions, the extra detail provided by PCR-SSP on the alleles carried by these patients was due to the failure of serology to differentiate the splits of DR3 and DR6 (table 2). The other two cases were due to the fact that although the splits can now be determined by serology, the antisera to do this were not commonly available when the patients presented in 1987 (DR5 patient) and 1988 (DR2 patient).

For $14 \%$ of patients at least some second round PCR reactions had to be repeated. More than $50 \%$ of these were because of failed control reactions in one or more tubes for patients who were actually homozygous. It is not possible for the serological typings to say how many fresh blood samples, extra B cell separations, or repeat typing reactions were necessary as no records had been kept.

\section{Discussion}

Our study has shown that within a routine hospital tissue typing laboratory, PCR-SSP is more accurate than serology for DRB1 typing. Because of the design of the study, the $11 \cdot 8 \%$ serology failure rate perhaps has limited value as an actual measure of the improvement that is possible. This laboratory is currently above the United Kingdom average for the accuracy of its serological tissue typing as measured by the National External Quality Assurance Scheme, so some laboratories might expect to see a greater improvement in their accuracy if they matched or surpassed our PCR-SSP performance.

We might equally be overestimating the advantage to be gained by PCR-SSP. Although there have been major improvements in B cell separation and antisera specificity since many of the serotypings were performed, several of the difficulties which were encountered still remain. In our laboratory, although the presence of a second allele has been suspected, DRB1*0103 has not been successfully assigned by serology. Both $\mathrm{DRB} 1{ }^{\star} 1303$ and ${ }^{\star} 1305$ have been misassigned as DR5 by serology, probably because of their unusual DQw1 association. The differentiation of DR4 and DR9 is still difficult in our experience, as can be the defining of a DR6 split.

Almost $40 \%$ of the patients had originally been typed before the introduction of Dynabeads to improve B cell separation. None had been retyped until this study. Serological retyping of case 11 (table 1) showed that both class I and class II antigens had originally been wrongly assigned. We suggest that as methods of tissue typing improve in accuracy, so data should be rechecked. As time on haemodialysis increases, decreased B cell viability and the concomitant problems in cell separation can make serology more difficult. ${ }^{16}$ There is no such problem for DNA typing. We have not only easily obtained PCR quality DNA from the blood of patients receiving long term haemodialysis, but also from blood samples stored for up to eight weeks at $4^{\circ} \mathrm{C}$.

Some of the discrepancies in tissue type which we have brought to light may have a more serious consequence for the patient than others. Cases 8 and 11 had errors involving DR6 and we also found failure to assign DR6 by serology in three of 27 other patients recently typed by both methods. Such mistakes are potentially very important for the kidney recipient as there is some suspicion that this antigen has a major influence on the success of these transplants. ${ }^{18} 19$

The advantages of genotyping over traditional serology are well documented. ${ }^{12}$ Patients' DNA can also be stored. This will allow rechecking without the need to recall patients and open the way for retrospective studies. Contrary to earlier reports ${ }^{20}$ we calculate genotyping, as performed in our laboratory, to be about two thirds the cost of serology.

The two PCR-SSP procedures have different benefits. The main advantage of the Olerup and Zetterquist PCR-SSP is speed: two hours from blood sample to result, compared to about $4 \frac{1}{2}$ hours for the method of Bein et al. Its main disadvantage is cost. Both 
the capital cost of a second generation thermocycler, on which the system was optimised, and its special reaction tubes are considerably higher than for a first generation machine. Adapting cycling parameters from one sort of PCR machine to another is not always easy. We do not obtain consistent results with the Olerup and Zetterquist primers, particularly the control set, on our machine under a variety of cycling regimens.

Both PCR-SSP methods have other drawbacks. They demand a thermocycler and submarine gel electrophoresis tank not normally available in a routine laboratory, and need two dedicated sets of micropipettes. This will not long remain merely tissue typing equipment, as more and more new PCR applications continue to be reported. Because molecular biology techniques are not yet familiar in routine hospital laboratories, the confidence necessary to use them "on call" may take time to develop. Cross-reactions (as in case 2) are not infrequent. In most cases they are relatively weak and easily discounted, but in homozygous patients or where the reaction is strong there is a danger that an allele will be wrongly assigned. Retesting suspected false positive results using a two-step PCR cycle of $94^{\circ} \mathrm{C}$ and $72^{\circ} \mathrm{C}$ for 30 seconds each, overcomes the problem. The "failure" of the PCR with one patient has been resolved by the inclusion of new primer mixes to amplify otherwise undetectable DR6 alleles. ${ }^{17}$ While genotyping is the most accurate way of defining tissue type as currently understood, and PCR-SSP the most suitable for the everyday demands of the routine laboratory, it will only remain so if it is constantly updated to take account of newly described alleles.

1 Marsh SGE, Bodmer JG. HLA class II nucleotide sequences, 1992. Tissue Antigens 1992;40:229-43.

2 Opelz G. HLA matching should be used for improving kidney transplant success rates. Transplant Proc 1991;
23:46-50.

3 Leivestad T, Berger L, Thorsby E. Beneficial effect of DR matching on cadaveric renal graft survival Scandiatransplant. Transplant Proc 1992;24:2447-8.

4 Schanz D, Blümke M, Keller E, Tanzi-Fetta RF, Kirste G. Influence of HLA matching on the outcome of kidney transplants in a single center analysis. Transplant Proc 1992;24:2455.

5 Bidwell J. DNA-RFLP analysis and genotyping of HLADR and DQ antigens. Immunology Today 1988;9:18-23.

6 Ivinson A, Sinnott PJ, Sheldon MS, Martin S, Dyer PA. Definition of HLA class II genotypes and phenotypes for matching solid organ donors and their recipients. Transplant Proc 1992;24:2480.

7 Vaughan RW, Lanchbury JSS, Marsh SGE, Hall MA, Bodmer JG, Welsh KI. The application of oligonucleotide probes to HLA Class II typing of the DRB subregion. Tissue Antigens 1990;36:149-55.

8 Buyse I, Decorte R, Baens M, et al. Rapid DNA typing of class II HIA antigens using the polymerase chain reaction and reverse dot blot hybridisation. Tissue Antigen 1993;41:1-14

9 Yunis I, Salazar M, Yunis EJ. HLA-DR generic typing by AFLP. Tissue Antigens 1991;38:78-88.

10 Bein G, Gläser R, Kirchner H. Rapid HLA-DRB1 genotyping by nested PCR amplification. Tissue Antigen 1992;39:68-73.

11 Olerup O, Zetterquist H. HLA-DR typing by PCR amplification with sequence-specific primers (PCR-SSP) in 2 hours: An alternative to serological DR typing in clinical practice including donor-recipient matching in cadaveric practice including donor-recipient matching in cad

12 Mickelson E, Smith A, McKinney S, Anderson G, Hansen JA. A comparative study of HLA-DRB1 typing by standard serology and hybridisation of non-radioactive sequence-specific oligonucleotide probes to PCR-amplified DNA. Tissue Antigens 1993;41:86-93.

13 Papola F, Valeri M, Torlone N, et al. Comparison between HLA-DR serologic typing and oligotyping in kidney transplant: A single center experience. Transplant Proc 1993;25:2239-40.

14 Tiercy J-M, Goumaz C, Mach B, Jeannet M. Application of HLA-DR oligotyping to 110 kidney transplant patients with doubtul serological typing. Transplantation 1991; 51:1110-14.

15 Mytilineos J, Scherer S, Dunkley H, et al. DNA HLA-DR typing results of 4000 kidney transplants. Transplantation typing results of 400

16 Mytilineos J, Scherer S, Opelz G. Comparison of RFLPDR beta and serological HLA-DR typing in 1500 individuals. Transplantation 1990;50:870-3.

17 Lynas C, Hurlock NJ, Copplestone JA, Prentice AG. PCR-SSP: A cautionary tale and an update of the Bein primer mixes. Tissue Antigens 1994;43:403-7.

18 Correla AM, Gomes da Costa A, Reis P, et al. DR6 compatibility and kidney graft survival. Transplant Proc 1992;24:2472.

19 Adam K, Polymenedis Z, Papakyriazi L, et al. HLA-A, B DR antigens and renal transplantation from cadaveric donors and living-related donors in Greek patients. donors and living-related donors

20 Wetzsteon PJ, Head MA, Fletcher LA, Norman DJ. Wetzsteon PJ, Head MA, Fletcher LA, Norman DJ
Confidence levels assigned to serologic HLA-DR typing predict DNA HIA-DR typing discrepancies. Transplant Proc 1992;24:2483-4. 